\title{
In vitro evaluation of a combination treatment involving anticancer agents and an aurora kinase $B$ inhibitor
}

\author{
SENNA SAKAI $^{1,2}$, HIROTO IZUMI ${ }^{1}$, YUKIKO YOSHIURA ${ }^{1}$, YOSHIFUMI NAKAYAMA ${ }^{3}$, \\ TAKAHIRO YAMAGUCHI ${ }^{4}$, YOSHIKAZU HARADA ${ }^{5}, \mathrm{CHIHO} \mathrm{KOI}^{6}$, \\ HIROYUKI KURATA $^{2}$ and YASUO MORIMOTO ${ }^{1}$
}

\begin{abstract}
${ }^{1}$ Department of Occupational Pneumology, Institute of Industrial Ecological Science, University of Occupational and Environmental Health School of Medicine, Kitakyushu 807-8555; ${ }^{2}$ Department of Bioscience and Bioinformatics, Biomedical Informatics R\&D Center, Kyushu Institute of Technology, Iizuka, Fukuoka 820-8502;

${ }^{3}$ Department of Gastroenterological and General Surgery, Wakamatsu Hospital, University of Occupational and Environmental Health School of Medicine, Kitakyushu 808-0024; ${ }^{4}$ Department of Hematology, University of Occupational and Environmental Health; ${ }^{5}$ Department of Dentistry and Oral Surgery, University Hospital, University of Occupational and Environmental Health; ${ }^{6}$ Department of Obstetrics and Gynecology, University of Occupational and Environmental Health School of Medicine, Kitakyushu 807-8555, Japan
\end{abstract}

Received August 1, 2015; Accepted August 25, 2016

DOI: $10.3892 / 01.2016 .5156$

\begin{abstract}
Aurora kinase B (AURKB) inhibitors are regarded as potential molecular-targeting drugs for cancer therapy. The present study evaluated the cytotoxic effect of a combination of AZD1152-hQPA, an AURKB inhibitor, and various anticancer agents on the HeLa human cervical cancer cell line, as well as its cisplatin-resistant equivalent HCP4 cell line. It was demonstrated that AZD1152-hQPA had an antagonistic effect on the cytotoxicity of cisplatin, etoposide and doxorubicin, but had a synergistic effect on that of all-trans-retinoic acid (ATRA), Am80 and TAC-101, when tested on HeLa cells. Cisplatin, etoposide and doxorubicin were shown to increase the cellular expression of AURKB, while ATRA, Am80 and TAC-101 downregulated its expression. These results suggested that AURKB expression is regulated by these anticancer agents at the transcriptional level, and that the level of expression of AURKB may influence the cytotoxic effect of AZD1152-hQPA. Therefore, when using anticancer agents, decreasing the expression of AURKB using a molecular-targeting drug may be an optimal therapeutic strategy.
\end{abstract}

Correspondence to: Dr Hiroto Izumi, Department of Occupational Pneumology, Institute of Industrial Ecological Science, University of Occupational and Environmental Health School of Medicine, 1-1 Iseigaoka, Yahatanishi-ku, Kitakyushu 807-8555, Japan E-mail: h-izumi@med.uoeh-u.ac.jp

Key words: aurora kinase B, aurora kinase B inhibitor, anticancer agent, combination treatment, combination index

\section{Introduction}

The human aurora kinase (AURK) family consists of three genes, including AURKA, AURKB and AURKC. Their gene products are located in different parts of the nucleus and have been suggested to function independently during the mitotic phase (M-phase) of the cell cycle (1-3). AURKB appears in the nucleus at the initial synthesis phase, and is involved in the regulation of cytokinesis by binding to several proteins containing the inhibitor Survivin $(4,5)$. It has been reported that AURKs are overexpressed in tumor cells and, therefore, they are thought to be potential molecular targets for the treatment of malignant tumors (6-9). A number of inhibitors of AURK (ZM447439, VX-680, AT9283, AZD1152, MLN8054 and MLN8237) have been developed (6). These agents inhibit AURKA and AURKB to varying degrees, and some are currently in phase I clinical trials (10). The previously described inhibitor AZD1152 is a prodrug that changes to the active form AZD1152-hQPA in the cytoplasm, which has a dominant effect on AURKB (11).

Chemotherapy using anticancer drugs, such as platinum-based therapies or taxanes, and radiotherapy are the most commonly employed strategies for the treatment of gynecological malignant tumors $(12,13)$. AURK inhibitors are thought to be an effective molecular-targeting drug for gynecological malignant tumors (6), and clinical trials for their use against leukemia and other cancers are underway (10). In the future, there is a possibility that ARUK inhibitors may be used in combination with anticancer agents. However, it is unknown which anticancer agents would function most effectively in combination with AURK inhibitors. Sun et al (14) reported that the AURKB inhibitor, VX-680, downregulated nuclear factor $(\mathrm{NF})-\kappa \mathrm{B}$ expression and increased the sensitivity of tumor cells to anticancer agents. Therefore, evaluation of the cellular expression or activity of $N F-\kappa B$ may emerge as an important basis for the use of AURKB inhibitors. 
Previously, we reported that cisplatin-resistant HCP4 cells, which are derived from the HeLa human cervical cancer cell line, overexpressed AURKB. Furthermore, when treated with AZD1152-hQPA, an AURKB inhibitor, the colony formation activity of cisplatin-resistant cells was shown to be significantly decreased, as compared with HeLa cells (15). Based on these results, it was hypothesized that a combination of cisplatin and molecular-targeting drugs may have a synergistic cytotoxic effect on malignant tumor cells. However, the present study demonstrated that an AURKB-specific small interfering RNA (siRNA) and AZD1152-hQPA antagonized the cytotoxic effect of cisplatin, whereas it had a synergistic effect on all-trans-retinoic acid (ATRA) and synthetic retinoids. These two different effects were thought to be due to differences in the expression levels of AURKB induced by treatment with specific anticancer agents. The present study aimed to investigate the expression levels of AURKB in the HeLa and HCP4 human cervical cancer cells lines, and propose a strategy for combination therapy involving AURKB inhibitors and anticancer agents.

\section{Materials and methods}

Cell culture. HeLa cells and their derived cisplatin-resistant HCP4 cells were established and kindly gifted by Professor Shin-Ichi Akiyama (Department of Molecular Oncology, Graduate School Medical and Dental Science, Kagoshima University, Kagoshima, Japan) (15). Both cell lines were cultured in RPMI 1640 Medium, GlutaMAX ${ }^{\mathrm{TM}}$ supplement (Invitrogen; Thermo Fisher Scientific, Inc., Waltham, MA, USA) containing 10\% fetal bovine serum (HyClone; GE Healthcare Life Sciences, Logan, UT, USA) in $5 \% \mathrm{CO}_{2}$ at $37^{\circ} \mathrm{C}$.

Antibodies and anticancer agents. Rabbit anti-AURKB (cat. no. 1788-1) and mouse anti- $\beta$-actin (cat. no. sc-47778) monoclonal antibodies were purchased from Epitomics (Burlingame, CA, USA) and Santa Cruz Biotechnology, Inc. (Santa Cruz, CA, USA), respectively. Cisplatin, etoposide and ATRA were purchased from Sigma-Aldrich; Merck Millipore (Darmstadt, Germany). Doxorubicin was obtained from Kyowa Hakko Kogyo, Co., Ltd. (Tokyo, Japan). AZD1152-hQPA was purchased from Chemietek (Indianapolis, IN, USA); it was dissolved in dimethyl sulfoxide to a concentration of $10 \mathrm{mM}$ and stored at $-20^{\circ} \mathrm{C}$. Synthetic retinoids, Am80 (Tamibarotene) and TAC-101 were kindly gifted by Dr Shudo Koichi of the Research Foundation ITSUU Laboratory (Tokyo, Japan).

Knockdown analysis using siRNAs. Knockdown of AURKB in HeLA cells was performed using AURKB-specific siRNA, as described previously (15). The following 25-bp double-stranded RNA oligonucleotides were commercially generated (Invitrogen; Thermo Fisher Scientific, Inc.): AURKB-specific siRNA,: 5'-UUUAGGUCCACCUUGACGAUGCGGC-3' and 5'-GCCGCAUCGUCA AGGUGGACCUAAA-3'. A total of 200 pmol siRNA was mixed with $5 \mu$ l Lipofectamine 2000 (Qiagen GmbH, Hilden, Germany) according to the manufacturer's protocol. After $20 \mathrm{~min}, 5 \times 10^{5}$ cells were gently mixed and incubated for additional $20 \mathrm{~min}$. Transfected cells were used for western blotting and colony formation assay. Negative control siRNA was purchased from Invitrogen (Thermo Fisher Scientific, Inc.).

Cell viability assays and combined effects of anticancer agents. Briefly, HeLa cells $\left(1 \times 10^{3}\right)$ were seeded into 96-well plates for $24 \mathrm{~h}$, and subsequently, cisplatin, etoposide, doxorubicin, ATRA, Am80, TAC-101 and AZD1152-hQPA were added to the cells at a maximum concentration at 20,40, 1, 500,100, 200 and $100 \mu \mathrm{M}$, respectively, and 2-fold serial dilutions were performed. For combination treatment, fixed combination ratios and 2-fold serial dilutions were employed. For AURKB, specific siRNA-transfected HeLa cells $\left(2 \times 10^{3}\right)$ were seeded into 96 -well plates at $24 \mathrm{~h}$ post-transfection, and the above indicated single agents were added to the cells with 2 -fold serial dilutions. After $72 \mathrm{~h}$, the surviving cells were stained with the water-soluble tetrazolium salt- 8 (TetraColor ONE; Seikagaku Corporation, Tokyo, Japan) for $2-3 \mathrm{~h}$ at $37^{\circ} \mathrm{C}$, according to the manufacturer's protocol. The absorbance was then measured at $450 \mathrm{~nm}$. To measure the half maximal inhibitory concentration $\left(\mathrm{IC}_{50}\right)$ in each experiment, CalcuSyn software version 2.0 (Biosoft, Cambridge, UK) was used. To evaluate the synergism or antagonism of the combination of an anticancer agent and AZD1152-hQPA, HeLa cells were treated with an anticancer agent alone, AZD1152-hQPA alone, or a fixed combination ratio of the anticancer agent and AZD1152-hQPA, as decided by the $\mathrm{IC}_{50}$ values. The experiments were performed in duplicate, with 2-fold serial dilutions. To assess whether there was a synergistic effect on cytotoxicity, the combination index (CI) was calculated using CalcuSyn software version 2.0. This method enables the quantification of synergism $(\mathrm{CI}<1)$ and antagonism $(\mathrm{CI}>1)$ at different concentrations and effect levels (16). Based on the median effective dose $(\mathrm{ED})_{50}, \mathrm{ED}_{75}$ and $\mathrm{ED}_{90}$ of the drug combinations, isobolograms were generated and synergy was evaluated using CalcuSyn software version 2.0.

Western blot analysis. Preparation of whole cell lysates and western blot analysis were performed as described previously (15). The cells were washed with PBS twice, and then lysed in buffer containing $50 \mathrm{mmol} / \mathrm{l}$ Tris- $\mathrm{HCl}(\mathrm{pH} 8.0)$, $1 \mathrm{mmol} / \mathrm{l}$ EDTA, $120 \mathrm{mmol} / \mathrm{l} \mathrm{NaCl}, 0.5 \%$ (v/v) Nonidet P-40, $10 \%$ (v/v) glycerol, $1 \mathrm{mmol} / 1$ phenylmethylsulfonyl fluoride and $1 \mathrm{mmol} / \mathrm{l}$ dithiothreitol. The lysates were centrifuged at $21,000 \times \mathrm{g}$ for $10 \mathrm{~min}$ at $4^{\circ} \mathrm{C}$, and the supernatants $(50 \mu \mathrm{g})$ were separated by $10 \%$ SDS-PAGE and then transferred onto polyvinylidene difluoride membranes. The blotted membranes were treated with $3 \%$ (w/v) skimmed milk in $10 \mathrm{mmol} / \mathrm{l}$ Tris, $150 \mathrm{mmol} / 1 \mathrm{NaCl}$ and $0.2 \%$ (v/v) Tween 20, and then incubated for $1 \mathrm{~h}$ at room temperature with the corresponding primary antibodies (1:1,000 dilution of rabbit anti-AURKB and $1: 10,000$ dilution of mouse anti- $\beta$-actin). Next, the membranes were then incubated for $40 \mathrm{~min}$ at room temperature with a peroxidase-conjugated secondary antibody [anti-rabbit immunoglobulin (Ig) G, peroxidase-linked species-specific whole antibody from donkey (NA934; GE Healthcare Life Sciences, Chalfont, UK) and anti-mouse IgG, peroxidase-linked speciesspecific whole antibody from sheep (NA931; GE Healthcare Life Sciences)] at 1:7,500 dilution. The bound antibody was visualized using an enhanced chemiluminescence kit (GE Healthcare Bio-Sciences, Pittsburgh, PA, USA), and the 
Table I. Evaluation of $\mathrm{IC}_{50}$.

\begin{tabular}{lrrr}
\hline Drug & HeLa IC & HCP4 IC $_{50}$ & IC $_{50}$ ratio $^{\mathrm{a}}$ \\
\hline Cisplatin $(\mu \mathrm{M})$ & $1.87 \pm 0.07$ & $65.53 \pm 0.83$ & 35.0 \\
Etoposide $(\mu \mathrm{M})$ & $9.72 \pm 0.14$ & $15.84 \pm 0.21$ & 1.6 \\
Doxorubicin $(\mathrm{nM})$ & $87.75 \pm 0.09$ & $146.74 \pm 7.04$ & 1.7 \\
ATRA $(\mu \mathrm{M})$ & $89.43 \pm 0.22$ & $84.34 \pm 1.31$ & 0.9 \\
Am80 $(\mu \mathrm{M})$ & $14.66 \pm 0.18$ & $14.82 \pm 0.48$ & 1.0 \\
TAC-101 $(\mu \mathrm{M})$ & $34.12 \pm 1.20$ & $38.75 \pm 0.36$ & 1.1 \\
AZD1152-hQPA $(\mu \mathrm{M})$ & $14.77 \pm 0.42$ & $31.22 \pm 0.15$ & 2.1 \\
\end{tabular}

${ }^{\mathrm{a}} \mathrm{IC}_{50}$ ratio $=\mathrm{IC}_{50}$ of $\mathrm{HCP} 4$ cell $/ \mathrm{IC}_{50}$ of $\mathrm{HeLa}$ cell. $\mathrm{IC}_{50}$, half-maximal inhibitory concentration; ATRA, all-trans-retinoic acid.

Table II. Fixed combination ratio.

\begin{tabular}{lcc}
\hline & \multicolumn{2}{l}{ Fixed combination ratio } \\
\cline { 2 - 3 } Drug combination & HeLa cells & HCP4 cells \\
\hline Cisplatin/AZD1152-hQPA & $1: 5$ & $2: 1$ \\
Etoposide/AZD1152-hQPA & $2: 5$ & $1: 2$ \\
Doxorubicin/AZD1152-hQPA & $1: 200$ & $1: 100$ \\
ATRA/AZD1152-hQPA & $5: 1$ & $3: 1$ \\
Am80/AZD1152-hQPA & $1: 1$ & $1: 2$ \\
TAC-101/AZD1152-hQPA & $2: 1$ & $1: 1$ \\
\hline
\end{tabular}

ATRA, all-trans-retinoic acid.

signal intensity was quantitated using Multi Gauge software version 3.0 (Fujifilm, Tokyo, Japan).

Reverse transcription-quantitative polymerase chain reaction $(R T-q P C R)$. Total RNA was purified from the transfected- and non-transfected HeLa cells using the RNeasy Mini kit (Qiagen, Inc., Valencia, CA, USA), and RT-qPCR was performed as described previously (17). Briefly, RT from total messenger RNA (mRNA) with random primers (Applied Biosystems; Thermo Fisher Scientific, Inc.) was performed with the High-Capacity cDNA Reverse Transcription kit (Thermo Fisher Scientific, Inc.), and then PCR with TaqMan ${ }^{\circledR}$ Universal Master Mix II with UNG (Thermo Fisher Scientific, Inc.) was conducted on the StepOnePlus ${ }^{\mathrm{TM}}$ Real-Time PCR System (Applied Biosystems; Thermo Fisher Scientific, Inc.) using the following cycling parameters: $2 \mathrm{~min}$ at $95^{\circ} \mathrm{C}$, followed by 40 cycles of $15 \mathrm{sec}$ at $95^{\circ} \mathrm{C}$ and $1 \mathrm{~min}$ at $60^{\circ} \mathrm{C}$, according to the manufacturer's protocol. The following primer sets for PCR were used: Hs01060665_g1 for $\beta$-actin, Hs00945858_g1 for AURKB and Hs01582072_m1 for AURKA (Applied Biosystems; Thermo Fisher Scientific, Inc.). The comparative $\mathrm{Cq}$ method was used to quantify the gene expression (18). Values were normalized to those for human $\beta$-actin. All samples were analyzed in duplicate in each experiment.

Statistical analysis. Student's t-tests were performed for statistical analysis of the variables between the two groups with
Table III. CI values for various concentrations of anticancer agents.

\begin{tabular}{lccccccr}
\hline & \multicolumn{3}{c}{ HeLa CI } & & \multicolumn{3}{c}{ HCP4 CI } \\
\cline { 2 - 3 } \cline { 7 - 8 } Drug & $\mathrm{ED}_{50}$ & $\mathrm{ED}_{75}$ & $\mathrm{ED}_{90}$ & & $\mathrm{ED}_{50}$ & $\mathrm{ED}_{75}$ & $\mathrm{ED}_{90}$ \\
\hline Cisplatin & 1.35 & 1.46 & 1.62 & 0.95 & 94.11 & 56,116 \\
Etoposide & 0.93 & 1.13 & 2.44 & 1.57 & 0.44 & 0.22 \\
Doxorubicin & 1.18 & 1.56 & 2.48 & & 1.71 & 0.66 & 0.34 \\
ATRA & 0.36 & 0.46 & 0.77 & & 0.88 & 0.50 & 5.44 \\
Am80 & 0.54 & 0.41 & 0.41 & & 0.55 & 0.28 & 2.74 \\
TAC-101 & 0.52 & 0.39 & 0.46 & 0.83 & 0.61 & 23.09 \\
\hline
\end{tabular}

CI, combination index; ATRA, all-trans-retinoic acid; $\mathrm{ED}_{50}$, median effective dose.

GraphPad StatMate statistical software version IV (GraphPad Software, Inc., La Jolla, CA, USA). Data are presented as the mean \pm standard deviation.

\section{Results}

AZD1152-hQPA antagonizes the cytotoxic effect of cisplatin on HeLa cells. In our previous study, we reported that the AURKB protein was overexpressed in cisplatin-resistant PCDP5 and HCP4 cells, as compared with the parent PC3 and HeLa cells, respectively, and was induced by cisplatin treatment in a concentration- and dose-dependent manner (15). Furthermore, the AURKB inhibitor, AZD1152-hQPA, sensitized cisplatin-resistant cells, but not parent cells, to cisplatin. To confirm whether cisplatin and AZD1152-hQPA have a synergistic cytotoxic effect on cancer cells, the present study calculated the CI using CalcuSyn software. First, the $\mathrm{IC}_{50}$ values of cisplatin and AZD1152-hQPA with HeLa cells were calculated (Table I), and were 1.87 and $14.8 \mu \mathrm{M}$, respectively. Based on these results, we employed a fixed combination ratio of cisplatin-to-AZD1152-hQPA of 1:5 (Table II), and treated HeLa cells with these agents using a single or a fixed combination concentration to calculate the CI. As is shown in Table III, the $\mathrm{CIs}$ at $\mathrm{ED}_{50}, \mathrm{ED}_{75}$ and $\mathrm{ED}_{90}$ were $1.35,1.46$ and 1.62, respectively, which suggested that, as the concentration of the drugs was elevated, the CI increased. These results indicated 
A
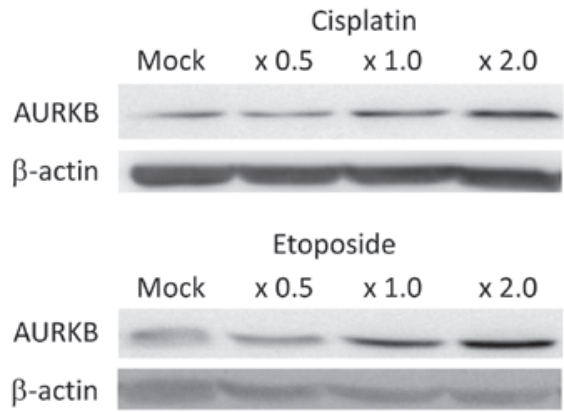

Doxorubicin

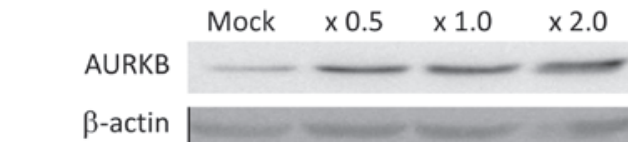

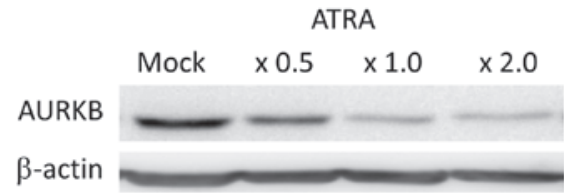

Am80

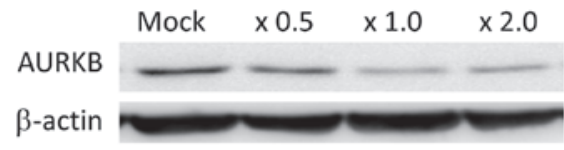

TAC-101

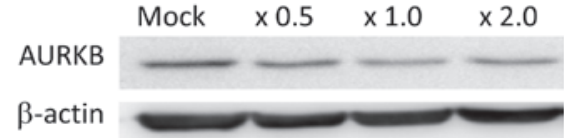

$\beta$-actin

B

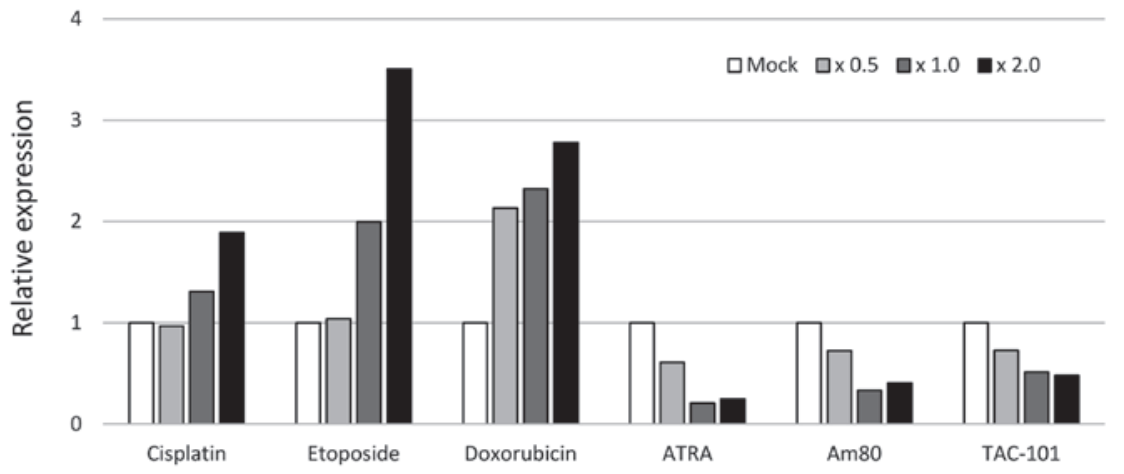

Figure 1. Protein expression of AURKB in HeLa cells is regulated by anticancer agents. (A) HeLa cells were treated with cisplatin, etoposide, doxorubicin, ATRA, Am80 or TAC-101 for $72 \mathrm{~h}$, and the protein expression of AURKB was analyzed by western blotting. $\mathrm{x} 0.5$, x1.0, and x2.0 indicate the amplification of the $\mathrm{IC}_{50}$ concentration shown in Table II. (B) Relative expression levels of AURKB were determined by densitometry. Each control was set to 1. AURKB, aurora kinase B; ATRA, all-trans-retinoic acid.

that cisplatin and AZD1152-hQPA did not have a synergistic cytotoxic effect, but an antagonistic cytotoxic effect, on HeLa cells.

Subsequently, the effect of the combined administration of cisplatin and AZD1152-hQPA on cisplatin-resistant HCP4 cells derived from HeLa cells was investigated. From the result of $\mathrm{IC}_{50}$ values for HCP4 (Table I), HCP4 cells were 35-times more resistant to cisplatin than HeLa cells. Therefore, a fixed combination ratio of cisplatin-to-AZD1152-hQPA of 2:1 was employed (Table II). As is shown in Table III, the CI of cisplatin and AZD1152-hQPA was very high, especially when used at $\mathrm{ED}_{75}$ and $\mathrm{ED}_{90}$, indicating that they had a strong antagonistic cytotoxic effect on HCP4 cells. The antagonistic cytotoxic effect on HCP4 cells was much higher than that of HeLa cells.

Effect of anticancer agents on AURKB expression. Consistent with our previous report (15), cisplatin treatment of HeLa cells induced the protein expression of AURKB in a concentration-dependent manner (Fig. 1). To confirm whether AURKB protein expression was transcriptionally induced by cisplatin, RT-qPCR was performed, and demonstrated that AURKB mRNA expression was upregulated by cisplatin (Fig. 2). It was hypothesized that the level of AURKB protein induced by anticancer agents might influence the cytotoxic effect of AZD1152-hQPA. Therefore, the effect of several anticancer agents on AURKB expression was examined.
Notably, etoposide and doxorubicin increased both the protein and mRNA expression levels of AURKB (Figs. 1 and 2). Conversely, ATRA, synthetic retinoids, Am80 and TAC-101 decreased the protein and mRNA expression levels of AURKB (Figs. 1 and 2). In addition, the mRNA expression levels of AURKA in HeLa cells treated with various anticancer agents were evaluated. Cisplatin, etoposide and doxorubicin increased the mRNA expression levels of AURKA, whereas ATRA, Am80 and TAC-101 decreased the mRNA expression levels of AURKA and AURKB (data not shown).

AURKB expression status is affected by anticancer agents, which influences the cytotoxic effect of AZD1152-hQPA. To investigate whether the AURKB expression status affects the cytotoxic effect of AZD1152-hQPA, CI values were calculated for the combined administration of AZD1152-hQPA with various anticancer agents. In HeLa cells, etoposide and doxorubicin, as well as cisplatin, increased the expression of AURKB and the CI value was $>1$ in all cases, with the exception of etoposide at $\mathrm{ED}_{50}$ (Table III). The highest CI for each agent was observed at $\mathrm{ED}_{90}$ for all cases. In contrast, the $\mathrm{CI}$ values for ATRA, Am80 and TAC-101 m, which decreased AURKB expression, was $<1$ for all cases. The CI value for ATRA gradually became higher as the concentration was increased; however, Am80 and TAC-101 retained low CI values, despite increased concentrations. In cisplatin-resistant HCP4 cells, the CI values of ATRA, Am80 and TAC-101 were 
Cisplatin

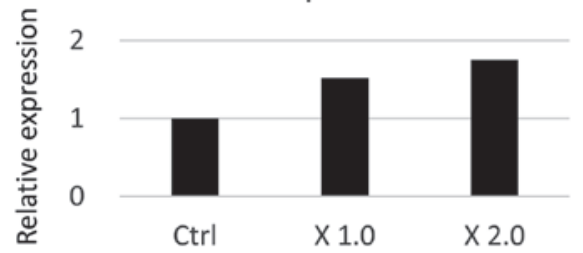

Etoposide

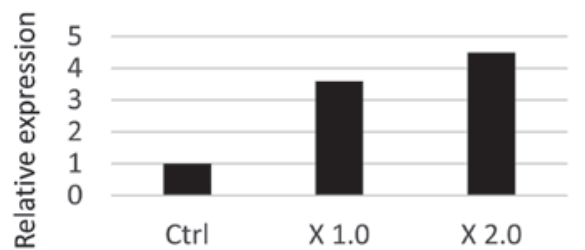

Doxorubicin

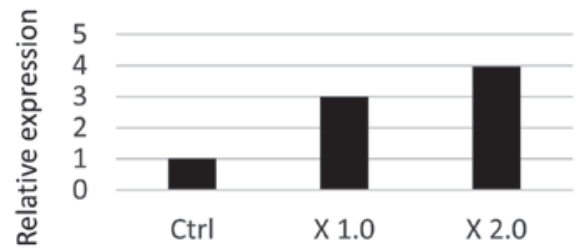

ATRA
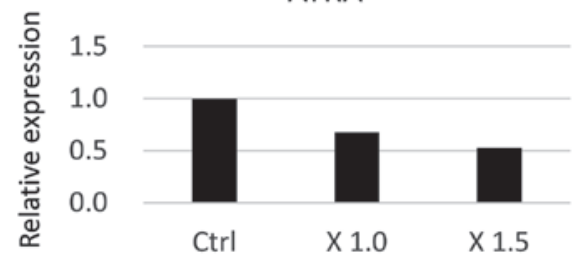

Am80

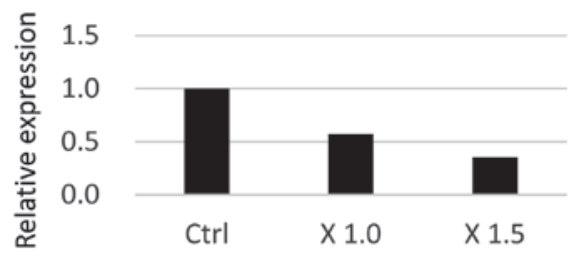

TAC-101

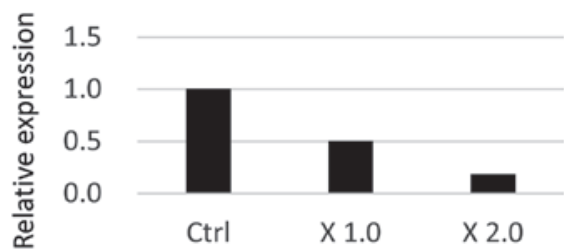

Figure 2. Aurora kinase B (AURKB) expression in HeLa cells is regulated by anticancer agents at the transcriptional level. HeLa cells were treated with cisplatin, etoposide, doxorubicin, ATRA, Am80 or TAC-101 for $72 \mathrm{~h}$, after which the mRNA expression levels of AURKB were analyzed by reverse transcriptionquantitative polymerase chain reaction. x1.0, x1.5 and x2.0 indicate the amplification of the $\mathrm{IC}_{50}$ concentration shown in Table II. ATRA, all-trans-retinoic acid.

A
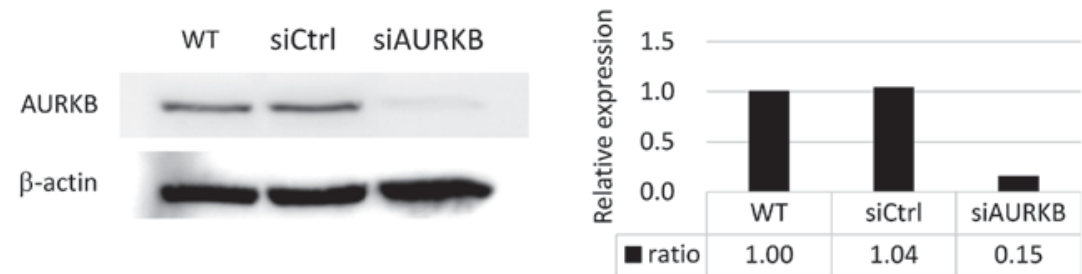

B

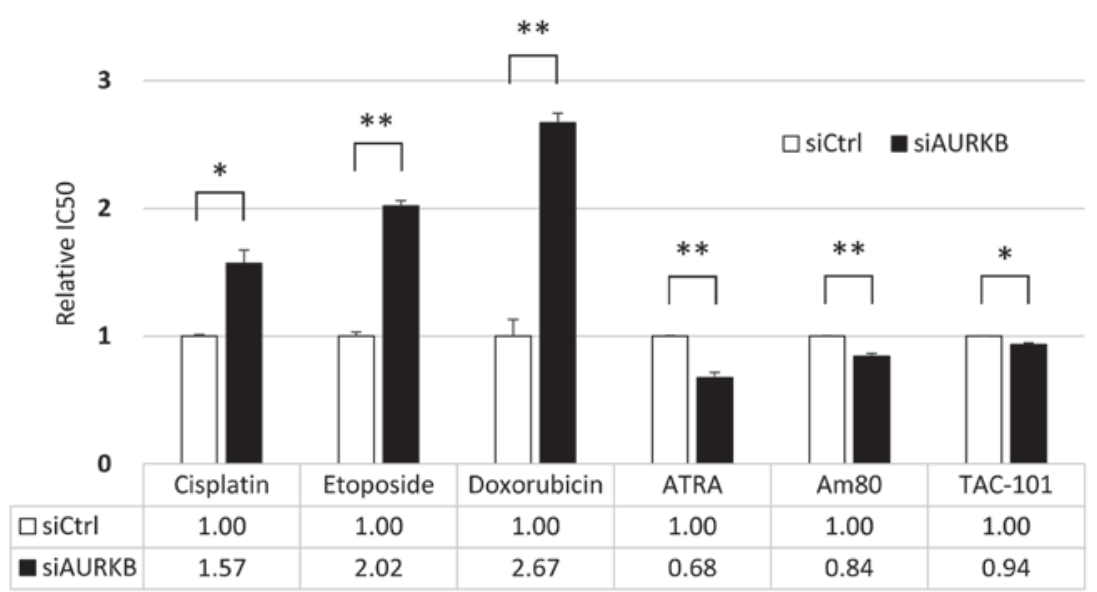

Figure 3. Knockdown of AURKB in HeLa cells and their sensitivity to anticancer agents. (A) HeLa cells were transfected with siCtrl or siAURKB for $48 \mathrm{~h}$, followed by treatment with various anticancer agents for $72 \mathrm{~h}$ and determination of AURKB expression levels by western blotting and densitometry. AURKB expression was normalized to $\beta$-actin, and the expression ratio of the WT cells was set as 1 . (B) Relative $\mathrm{IC}_{50}$ values were obtained from the concentration-response curves for each anticancer agent. The $\mathrm{IC}_{50}$ value for siCtrl-transfected cells was set to $1 .{ }^{*} \mathrm{P}<0.05$ and ${ }^{* * *} \mathrm{P}<0.01 \mathrm{vs}$. siCtrl-transfected cells. AURKB, aurora kinase B; ATRA, all-trans-retinoic acid; WT, wild-type; siCtrl, control small interfering RNA (siRNA); siAURKB, AURKB-specific siRNA; IC50, half-maximal inhibitory concentration.

$<1$ at $\mathrm{ED}_{50}$ and $\mathrm{ED}_{75}$, but were $>1$ at $\mathrm{ED}_{90}$ (Table III). Furthermore, the $\mathrm{CI}$ of etoposide and doxorubicin at $\mathrm{ED}_{75}$ and $\mathrm{ED}_{90}$ was $<1$ when tested using HeLa cells. As is shown in Table I, cisplatin-resistant HCP4 cells had a multidrug-resistant phenotype, but this mechanism had no effect on the cytotoxicity of ATRA and synthetic retinoids. 
Knockdown of AURKB and the cytotoxic effects of anticancer agents. AZD1152-hQPA decreases the activity of AURKB (11). To investigate whether knockdown of AURKB affected the cytotoxic effect of ATRA, Am80 and TAC-101, HeLa cells were transfected with AURKB-specific siRNA and treated with these agents. As is shown in Fig. 3A, the expression of AURKB was downregulated in AURKB-specific siRNA-knockdown HeLa cells, as compared with cells transfected with control siRNA. Knockdown of AURKB increased the $\mathrm{IC}_{50}$ values of cisplatin, etoposide and doxorubicin, and decreased the $\mathrm{IC}_{50}$ values of ATRA, Am80 and TAC-101, as compared with the control (Fig. 3B).

\section{Discussion}

AURKs are important molecules involved in mitosis, and AURKA and AURKB have been shown to be overexpressed in several types of cancer (1). Therefore, AURKs are potential novel molecular targets for the prevention of cancer proliferation, and clinical trials have been performed (10). It has been reported that AURKB inhibitors are able to enhance the cytotoxic effects of anticancer agents (19-21). Fu et al (19) reported that the AURK inhibitor VE 465 was able to enhance the antitumor activity of carboplatin in human ovarian cancer cells. Fiskus et al (20) used a combination treatment involving the pan-AURK inhibitor MK-0457 and vorinostat in human breast cancer cells, and demonstrated a synergistic effect in both in vitro and in vivo assays. Therefore, the present study aimed to investigate the cytotoxic effect of the AURKB-specific inhibitor, AZD1152-hQPA, and cisplatin on the HeLa cervical adenocarcinoma cell line, and demonstrated that the effects were antagonistic. Previously, we reported that the expression of AURKB was increased following treatment of cancer cells with cisplatin (15). It was hypothesized that the induction of AURKB by cisplatin treatment may alter the sensitivity of cancer cells to AZD1152-hQPA, and it was decided that we would search for anticancer agents that increased or decreased AURKB expression. In the present study, it was demonstrated that doxorubicin, etoposide and cisplatin increased the expression of AURKB, while ATRA, Am80 and TAC-101 decreased its expression. Subsequently, the combined effects of these anticancer agents with AZD1152-hQPA were investigated, their CIs were calculated, and it was demonstrated that the combinations that increased AURKB expression showed antagonistic cytotoxic effects on HeLa cells. In contrast, the combination of AZD1152-hQPA with anticancer agents that decreased the expression of AURKB showed synergistic cytotoxic effects on HeLa cells. These results indicated that AURKB expression may influence the cytotoxic effect of AZD1152-hQPA. To the best of our knowledge, there has been no previous report showing a synergistic cytotoxic effect for AZD1152-hQPA used in combination with anticancer agents. Zhang and Zhang (21) reported that ZM447439, an AURKB inhibitor, suppressed the growth of SiHa cervical cancer cells and enhanced their chemosensitivity to cisplatin, which was inconsistent with the results of the present study. Both AZD1152-hQPA and ZM447439 also inhibited AURKA, but the effect of AZD1152-hQPA on AURKB inhibition was greater than ZM447439 (6). Furthermore, AURKA expression was increased by cisplatin in the present study. This discrepancy might be due to differing specificities for AURKs or the type of cells used.

In the present study, cisplatin, doxorubicin and etoposide increased the cellular expression of AURKB, while ATRA, Am80 and TAC-101 decreased it. Notably, these anticancer agents regulated AURKB expression at the transcriptional level, as demonstrated by RT-qPCR. From sequence alignment analysis, the Alu sequence upstream of the transcription start site of the AURKB gene was identified, and it was observed that the promoter region contained $\sim 230$ bp (data not shown). the promoter region of the AURKB gene is $\sim 230$ bp (data not shown). Kimura et al (22) reported that the E2 family (E2F) of transcription factors promote the transcription of the AURKB gene via a cell-cycle-dependent element (CDE) in the promoter region. Ianari et al (23) reported that treatment with cisplatin or doxorubicin increased E2F-1 expression, and E2F-1 Ser403 phosphorylation was induced by doxorubicin (24). Conversely, E2F-1 expression, induced by treatment with estrogen, was inhibited by treatment with a trans-retinoic acid (25). Therefore, the CDE/E2F-1 pathway may be associated with AURKB gene expression and regulated by anticancer agents. Notably, the effects of anticancer agents on the cell cycle in previous studies were different. Cisplatin, doxorubicin and etoposide induced G2/M-phase cell cycle arrest (26-28), while ATRA, Am80 and TAC-101 induced G1 cell cycle arrest (29-31). Further analysis is required to elucidate the associations among AURKB expression and anticancer agents.

Previously, we reported that ARUKB expression in cisplatin-resistant HCP4 cells was upregulated, as compared with parent HeLa cells, and demonstrated that HCP4 cells were hypersensitive to AZD1152-hQPA using colony formation assays (15). However, in the present study, cytotoxicity assays demonstrated that HCP4 cells were resistant to AZD1152-hQPA. This discrepancy may be due to differences in the assays performed and the growth rates of each cell line. Furthermore, upregulation of AURKB expression in HCP4 cells may have contributed to induce resistance to AZD1152-hQPA. Unlike HeLa cells, the combined treatment of AZD1152-hQPA and anticancer agents had a biphasic effect in cisplatin-resistant $\mathrm{HCP} 4$ cells: the $\mathrm{ED}_{75}$ of ATRA and synthetic retinoids was synergistic, while the $\mathrm{ED}_{90}$ of these agents was antagonistic. This biphasic effect may also be influenced by the slow growth rate of HCP4 cells. Further analysis is required to develop an effective method to overcome cisplatin resistance when the combination of AURK inhibitors and anticancer agents is used.

In the present study, AZD1152-hQPA had an antagonistic effect on cisplatin. Therefore, whether AURKB knockdown was able to inhibit the cytotoxic effect of cisplatin was investigated. AURKB knockdown resulted in the resistance of HeLa cells to cisplatin, doxorubicin and etoposide, while it sensitized the cells to ATRA, Am80 and TAC-101, as well as AZD1152-hQPA. These results suggested that strategies involving AURKB inhibition or knockdown may have similar effects as anticancer agents.

In conclusion, the results of the present study suggested that a combination of molecular-targeting drugs against AURKB and anticancer agents may influence the cytotoxic effects in cells. The optimal combination therapy may be determined by revealing these mechanisms. 


\section{Acknowledgements}

This study was supported in part by Grants-in-Aid for Scientific Research from the Ministry for Education, Culture, Sports, Science and Technology of Japan (grant no. 24591987).

\section{References}

1. Kollareddy M, Dzubak P, Zheleva D and Hajduch M: Aurora kinases: Structure, functions and their association with cancer. Biomed Pap Med Fac Univ Palacky Olomouc Czech Repub 152: 27-33, 2008 .

2. Hochegger H, Hégarat $\mathrm{N}$ and Pereira-Leal JB: Aurora at the pole and equator: Overlapping functions of Aurora kinases in the mitotic spindle. Open Biol 3: 120185, 2013.

3. Vas AC and Clarke DJ: Aurora B kinases restrict chromosome decondensation to telophase of mitosis. Cell Cycle 7: 293-296, 2008.

4. Dutertre S, Descamps S and Prigent C: On the role of aurora-A in centrosome function. Oncogene 21: 6175-6183, 2002.

5. Carmena M, Ruchaud S and Earnshaw WC: Making the Auroras glow: Regulation of Aurora A and B kinase function by interacting proteins. Curr Opin Cell Biol 21: 796-805, 2002.

6. Umene K, Banno K, Kisu I, Yanokura M, Nogami Y, Tsuji K, Masuda K, Ueki A, Kobayashi Y, Yamagami W, et al: Aurora kinase inhibitors: Potential molecular-targeted drugs for gynecologic malignant tumors. Biomed Rep 1: 335-340, 2013.

7. Mehra R, Serebriiskii IG, Burtness B, Astsaturov I and Golemis EA: Aurora kinases in head and neck cancer. Lancet Oncol 14: e425-e435, 2013.

8. Baldini E, Sorrenti S, D'Armiento E, Prinzi N, Guaitoli E, Favoriti P, Gnessi L, Moretti C, Bianchini M, Alessandrini S, et al: Aurora kinases: New molecular targets in thyroid cancer therapy. Clin Ter 163: e457-e462, 2012.

9. Kelly KR, Ecsedy J, Mahalingam D, Nawrocki ST, Padmanabhan S, Giles FJ and Carew JS: Targeting aurora kinases in cancer treatment. Curr Drug Targets 12: 2067-2078, 2011.

10. Cheung CH, Sarvagalla S, Lee JY, Huang YC and Coumar MS: Aurora kinase inhibitor patents and agents in clinical testing: An update (2011-2013). Expert Opin Ther Pat 24: 1021-1038, 2014.

11. Mortlock AA, Foote KM, Heron NM, Jung FH, Pasquet G, Lohmann JJ, Warin N, Renaud F, De Savi C, Roberts NJ, et al: Discovery, synthesis, and in vivo activity of a new class of pyrazoloquinazolines as selective inhibitors of aurora B kinase. J Med Chem 50: 2213-2224, 2007.

12. Lorusso D, Petrelli F, Coinu A, Raspagliesi F and Barni S: A systematic review comparing cisplatin and carboplatin plus paclitaxel-based chemotherapy for recurrent or metastatic cervical cancer. Gynecol Oncol 133: 117-123, 2014.

13. Viswanathan AN: Advances in the use of radiation for gynecologic cancers. Hematol Oncol Clin North Am 26: 157-168, 2012 .

14. Sun C, Chan F, Briassouli P and Linardopoulos S: Aurora kinase inhibition downregulates NF-kappaB and sensitises tumour cells to chemotherapeutic agents. Biochem Biophys Res Commun 352: 220-225, 2007.

15. Akiyama M, Izumi H, Wang KY, Yamaguchi T, Kuma A, Kitamura N, Harada Y, Oya R, Yamaguchi K, Iwai Y and Kohno K: Hypersensitivity to aurora kinase inhibitors in cells resistant against platinum- containing anticancer agents. Anticancer Agents Med Chem 14: 1042-1050, 2014.
16. Reynolds $\mathrm{CP}$ and Maurer BJ: Evaluating response to antineoplastic drug combinations in tissue culture models. Methods Mol Med 110: 73-183, 2005.

17. Yamaguchi T, Kurita T, Nishio K, Tsukada J, Hachisuga T, Morimoto Y, Iwai Y and Izumi H: Expression of BAF57 in ovarian cancer cells and drug sensitivity. Cancer Sci 106: 359-366, 2015.

18. Livak KJ and Schmittgen TD: Analysis of relative gene expression data using real-time quantitative PCR and the 2(-Delta Delta C(T)) Method. Methods 25: 402-408, 2001.

19. Fu S, Li Y, Huang J, Liu T, Hong Z, Chen A, Bast RC, Kavanagh JJ, Gershenson DM, Sood AK and Hu W: Aurora kinase inhibitor VE 465 synergistically enhances cytotoxicity of carboplatin in ovarian cancer cells through induction of apoptosis and downregulation of histone 3. Cancer Biol Ther 13: 1034-1041, 2012.

20. Fiskus W, Hembruff SL, Rao R, Sharma P, Balusu R, Venkannagari S, Smith JE, Peth K, Peiper SC and Bhalla KN: Co-treatment with vorinostat synergistically enhances activity of Aurora kinase inhibitor against human breast cancer cells. Breast Cancer Res Treat 135: 433-444, 2012.

21. Zhang L and Zhang S: ZM447439, the Aurora kinase B inhibitor, suppresses the growth of cervical cancer SiHa cells and enhances the chemosensitivity to cisplatin. J Obstet Gynaecol Res 37: 591-600, 2011.

22. Kimura M, Uchida C, Takano Y, Kitagawa M and Okano Y: Cell cycle-dependent regulation of the human aurora B promoter. Biochem Biophys Res Commun 316: 930-936, 2004.

23. Ianari A, Gallo R, Palma M, Alesse E and Gulino A: Specific role for $\mathrm{p} 300 / \mathrm{CREB}$-binding protein-associated factor activity in E2F1 stabilization in response to DNA damage. J Biol Chem 279: 30830-30835, 2004.

24. Real S, Espada L, Espinet C, Santidrián AF and Tauler A: Study of the in vivo phosphorylation of E2F1 on Ser403. Biochim Biophys Acta 1803: 912-918, 2010.

25. Hartman J, Müller P, Foster JS, Wimalasena J, Gustafsson JA and Ström A: HES-1 inhibits 17beta-estradiol and heregulin-beta1-mediated upregulation of E2F-1. Oncogene 23: 8826-8833, 2004.

26. Sorenson CM, Barry MA and Eastman A: Analysis of events associated with cell cycle arrest at G2 phase and cell death induced by cisplatin. J Natl Cancer Inst 82: 749-755, 1990.

27. Nam C, Doi K and Nakayama H: Etoposide induces G2/M arrest and apoptosis in neural progenitor cells via DNA damage and an ATM/p53-related pathway. Histol Histopathol 25: 485-493, 2010.

28. Ling YH, el-Naggar AK, Priebe W and Perez-Soler R: Cell cycle-dependent cytotoxicity, G2/M phase arrest, and disruption of p34cdc2/cyclin B1 activity induced by doxorubicin in synchronized P388 cells. Mol Pharmacol 49: 832-841, 1996.

29. Wang JG, Barsky LW, Davicioni E, Weinberg KI, Triche TJ, Zhang XK and Wu L: Retinoic acid induces leukemia cell G1 arrest and transition into differentiation by inhibiting cyclin-dependent kinase-activating kinase binding and phosphorylation of PML/RARalpha. FASEB J 20: 2142-2144, 2006.

30. Nakazato T, Okudaira T, Ishikawa C, Nakama S, Sawada S, Tomita M, Uchihara JN, Taira N, Masuda M, Tanaka Y, et al: Anti-adult T-cell leukemia effects of a novel synthetic retinoid, Am80 (Tamibarotene). Cancer Sci 99: 2286-2294, 2008.

31. Fujimoto K, Hosotani R, Doi R, Wada M, Lee JU, Koshiba T, Miyamoto Y, Tsuji S, Nakajima S and Imamura M: Induction of cell-cycle arrest and apoptosis by a novel retinobenzoic-acid derivative, TAC-101, in human pancreatic-cancer cells. Int J Cancer 81: 637-644, 1999. 